\title{
Optimal Performance for Detection Systems in Wireless Passive Sensor Networks
}

\author{
Ashraf Tantawy, Xenofon Koutsoukos, and Gautam Biswas \\ Institute for Software Integrated Systems (ISIS) \\ Department of Electrical Engineering and Computer Science \\ Vanderbilt University, Nashville, TN, 37235, USA \\ Email: ashraf.tantawy, xenofon.koutsoukos, gautam.biswas@ vanderbilt.edu
}

\begin{abstract}
Passive wireless sensors have emerged as a new technology to measure a vast majority of phenomena in our daily life. Passive sensors require no power source, and therefore their application domains are numerous, including health care, infrastructure protection, and national security, among many others. The deployment of wireless passive sensors and their readers has changed how detection needs to be performed. Passive sensors cannot pre-process the measurements as they have limited computational power. Therefore, no local decision is taken. Also, the reader polls the information from multiple sensors at the same time, and this causes collisions and hence packet drops and delays. In this paper, we formulate the detection performance, with non-ideal channels, in a probabilistic way, and compare with classical detection performance. We design an optimal adaptive Neyman-Pearson detector, given the channel probabilistic model, by formulating and solving a constrained optimization problem.
\end{abstract}

Index Terms-Neyman-Pearson detection, intermittent observations, wireless passive sensor, ROC curve.

\section{INTRODUCTION}

In classical detection theory, statistical hypothesis testing is applied to detect noisy signals. The main problem is to design the optimal detector (according to some pre-defined criteria) that distinguishes between two or more hypotheses (sometimes referred to as phenomena or state of nature) given noisy observations. This problem has been studied extensively [1], and different detectors have been proposed. Examples are the Neyman-Pearson (NP) detector and the Maximum Likelihood (ML) detector [2]. Classical detection theory will be referred to as centralized detection hereafter.

The emergence of distributed radar has given another perspective for the detection problem, namely Decentralized Detection (DD). DD has been an active area of research during the 1980s and early 1990s. In DD, multiple sensors relay information (after pre-processing) to a fusion center, and the problem is to design both the optimal local decision rules and the fusion rule to detect events as accurately as possible [3].

Recently, Wireless Sensor Networks (WSNs) have been employed in DD applications, adding to the challenge of detection the issue of channel imperfection, which causes errors in the transmission. There is recent work that discusses the effect of non-ideal channels on the design of decentralized detectors [4], [5]. However, the main assumption is that the sensor nodes have sufficient computational power to pre- process the observations and take a decision. This assumption is no longer valid in the case of passive wireless sensors.

In the last few years, passive wireless sensors have appeared as a new technology for sensors that does not require any power source. The basic idea is to use a powered reader to poll the data from different passive sensors. The passive sensor uses the incident power from the reader to energize its local circuitry. A variety of ways exist for the sensor to modulate the reader incident wave and relay its information back to the reader. The most popular example for this architecture is RFID technology [6].

Motivated by RFID technology, passive wireless sensors have been developed to measure a vast majority of phenomena in our daily life. In the environmental monitoring domain, passive wireless sensors that detect bio-hazardous materials are proposed in [7]. An example of a passive wireless sensor for measuring temperature, stress, strain, acceleration and displacement using Surface Acoustic Wave (SAW) transducer is described in [8]. In the health care domain, a passive strainsensor technology for the measurement of small strains on bones or fixation systems in the human body is presented in [9]. In the automotive industry, passive wireless strain monitoring of car tires is described in [10].

The introduction of passive wireless sensors as a replacement for active sensors has changed how detection needs to be performed. The main change comes from the fact that a passive sensor has limited computational power and memory, and therefore, it cannot pre-process the measurements before transmission to the reader. Also, in a passive WSN architecture, the reader polls the information from multiple sensors at the same time, giving rise to collisions and hence packet drops and delays.

Passive wireless sensors communication adheres to strict industry standards. The standards deal with air interface protocols (the way tags and readers communicate), data content (the way data is formatted), and conformance (ways to test products). These are RFID standards, where passive sensors communicate arbitrary sensor data by emulating an RFID tag whose ID encodes the desired sensor data [11]. Conforming to standards guarantees inter-operability between different vendors' passive sensors and readers, and reduces sensor cost, which is the primary attractive feature for passive sensors, in addition to their battery-less operation. 
The fact that wireless sensors communicate using data network packets, is not adequately addressed in the typical work of DD in WSNs. This fact, although simple, has a great impact on the way the DD system is represented and analyzed. Typical work on DD in WSNs assumes a finite alphabet for local sensors, that are transmitted using orthogonal set of signals, and the objective is to study the effect of the channel (fading and noise) on the received waveforms [3], [12]. In typical WSNs, sensor data is submitted in a frame that includes header information, the error correcting code, in addition to other packet overhead. Effect of the channel on the received waveforms is of interest only to the detection task performed by the digital communication system at the physical layer level, to infer the transmitted symbol (representing a string of bits). As far as the sensor data (in the packet payload) is concerned, the data may not arrive, or arrive with arbitrary delay.

As an illustrating example, fading is one of the common phenomena affecting the transmitted signals [13]. In a data network, fading may cause an error in the received packet, which for all practical purposes, is detected at the receiver with very high probability. Therefore, the packet may be in error, and a retransmission may be requested, or the packet may arrive with no errors. Therefore, the effect of fading on the data network can be captured at a higher level, by modeling the data network in a probabilistic way.

In previous work, we studied the degradation in the detector performance as a result of channel imperfection [14]. We assumed a single passive sensor to reader communication over a Bernoulli channel, to model the missing observations effect of a typical data network. Since passive sensors lack computational power, raw observations, without pre-processing, are assumed to be sent directly to the reader. We obtained the detector performance both analytically and through Monte Carlo simulations, and proposed a heuristic approach to restore the detector performance, by increasing the number of samples, and hence increasing the delay for detection.

In this paper, we formalize the NP detector performance analysis with intermittent observations, in a probabilistic framework. We also formulate, and solve, the optimization problem to obtain the optimal design for the detector in the case of non-ideal channel. In contrast with our previous work, we do not put any restriction on the detector, such as existence of a sufficient statistic. Rather, we assume any detector with a general ROC curve. We also assume a generic probabilistic channel model and emphasize the mathematical difference between the detection problem in both the ideal and the nonideal channel cases.

The rest of the paper is organized as follows: In Section III, we formulate the detection problem using passive wireless sensors, with a non-ideal channel between the sensors and the detector. We explain the network effect on the detection system, and highlight the difference between this formulation and other formulations reported in the related work. We present some preliminary results from our previous work in Section IV. In Section V, we give a probabilistic interpre- tation for the performance of the detector with non-ideal channel, using Neyman-Pearson (NP) criterion. In Section VI, we formulate and solve the constraint optimization problem, resulting in an optimal adaptive detector design, working with intermittent observations. Finally, we conclude the work with future research directions in Section VII.

\section{RELATED WORK}

Classical detection theory, with particular attention to signal processing applications, is discussed in [1], [2]. The research on decentralized detection is largely attributed to the seminal work of Tenney and Sandell [15]. The optimal decision rules for the local nodes and the fusion center are derived under various problem settings and different optimality criteria. For a more comprehensive survey in this area, the readers are referred to [3], [5] and the references therein.

For DD under WSN constraints, fusion rules with fading channel, given fixed local decision rules, are studied in [12], with a problem revisit in [13] to relax the assumption of channel state information availability at the fusion center. The optimality of the Likelihood Ratio (LR) test for local sensor decisions, with non ideal channel, is proved in [4]. The detection and estimation performances, when the sensors and the fusion center communicate over multi-access fading channel are studied in [16], where it is assumed that the sensor transmits to the fusion center the type of the sensor observations. The variations in the false alarm and detection probabilities of a DD system due to the errors caused by the links between sensors and the fusion center are studied in [17], where a Rayleigh fading channel is assumed. The design of the optimal quantizer and fusion rule, using both Bayesian and Neyman-Pearson approaches, when the transmission is subject to noise and inter-channel interference is discussed in [18]. The distributed detection problem over Multiple Access Channel (MAC), as opposed to the traditional assumption of parallel access channel, is studied in [19].

The performance analysis for NP detectors, with non-ideal channel, is studied in [14]. A Bernoulli communication channel is assumed, and a closed form expressions are obtained for the performance metrics, provided a sufficient statistic exists for the data samples. The analysis is augmented by Monte Carlo Simulation studies, and an adaptive detector design is proposed, with the penalty of increasing the delay for detection. In this paper, as opposed to the previous work, where the performance analysis of the already designed detector is carried out, we derive the optimal performance that can be obtained given the probabilistic channel model.

\section{PROBLEM Formulation}

Figure 1 illustrates the detection system architecture. We consider the binary hypothesis testing problem where the state of nature is represented by one of two hypotheses, $\mathcal{H}_{0}$ and $\mathcal{H}_{1}$. Every passive sensor, $S_{i}$, obtains an observation vector, $\mathbf{x}_{i}$ from the environment, and employs a local decision rule, $g\left(\mathbf{x}_{i}\right)$, to make a decision $\mathbf{y}_{i}$. The local decision rule may widely vary from submitting the raw observations to 


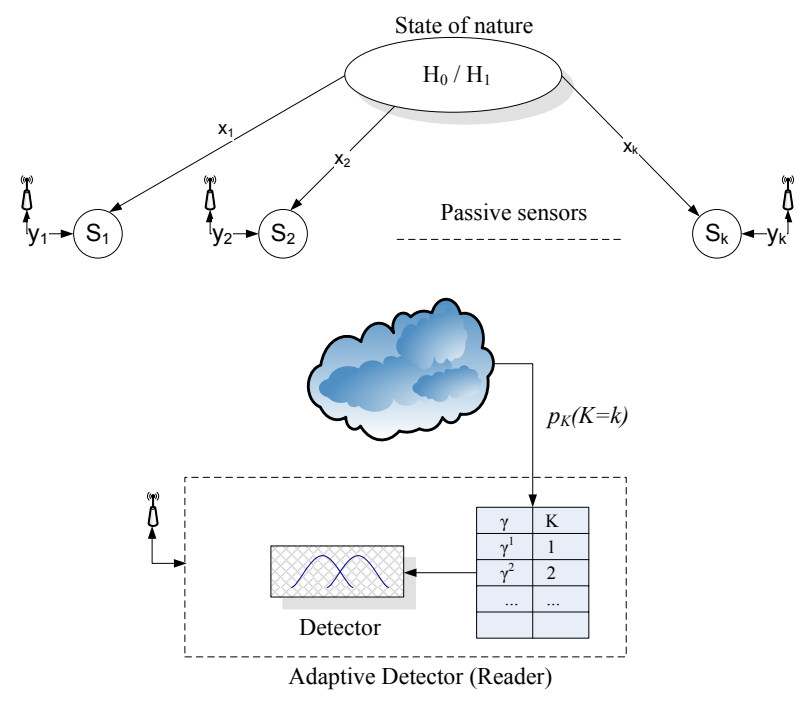

Fig. 1. Detection with Passive wireless sensors

submitting just a hard decision (event present/not present). For passive sensors, because of lack of computational power, the sensors submit the raw observations without preprocessing. Therefore, $\mathbf{y}_{i}=g\left(\mathbf{x}_{i}\right)=\mathbf{x}_{i}$. We further assume that sensor observations are Independent and Identically Distributed (IID) through space and time, so that the reader is using the observations received, for decision, without regard to their source.

The set of local observations are transmitted over an unreliable wireless channel to the reader. Since sensor data is sent using data network packets, we model the channel as a data network, capturing its effect on the transmitted packets.

Figure 2 illustrates the various effects of the communication channel, and how they map to only two data network effects, namely packet (observation) loss and observation delay. For example, a fading channel may cause the packet to be received in error. Being in error state, and depending on the communication protocol, a retransmission may be requested by the receiver. If the packet is retransmitted successfully after the retransmission request, then the final effect of the data network is an observation delay. If, on the other hand, the communication protocol is configured with a retransmission timeout, and the timeout elapsed, then the packet is considered dropped, and the final effect of the data network is an observation loss. By similar reasoning, all other channel effects shown are mapped to only two data network effects: packet delay and packet loss. In this discussion, we neglect the probability that a packet error will not be detected at the receiver. For modern digital communication systems, this probability is very small, and could be neglected without impact on the analysis [20].

In this paper, we assume only the missing observations effect of the data network. Assuming that the reader takes a decision every time period $T$, a reasonable probabilistic model for the channel would be defining the total number of samples received over the network, in that time period, as a random variable $K$, with a PDF $p_{K}(K=k ; T)$. Since the total

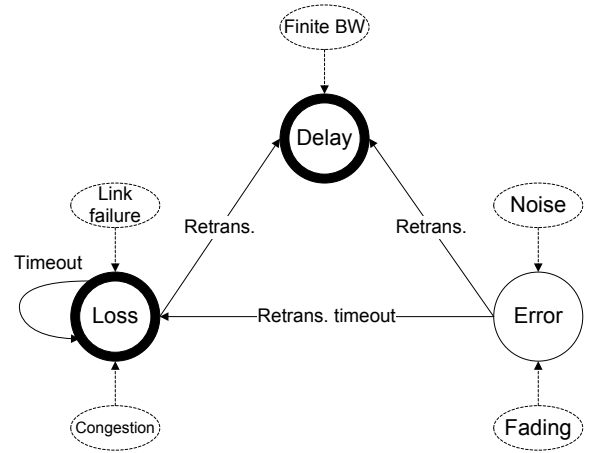

Fig. 2. Data network effect

number of samples carried by the communication channel is a function of other parameters, like channel capacity, we denote the PDF by $p_{K}(K=k ; \theta)$, assuming the vector $\theta$ captures all dependencies.

Upon receiving a subset of the observations (due to packet loss), the reader takes a binary decision $u$ (event present/not present). The reader has a probability of false alarm and detection, denoted by $P_{f}$ and $P_{d}$, respectively. The relationship between $P_{d}$ and $P_{f}$ defines the well-known Receiver Operating Characteristics (ROC) curve. More observations translate to better performance, and hence sharper-step ROC curve. Therefore, we have a family of ROC curves for different values of $K$. Also, for every ROC curve, we change the operating point by varying the detector threshold, $\gamma$. These facts are illustrated in Figure 3. We use the terms reader and detector interchangeably in this paper, since the detector has the additional role of polling the passive sensor data.

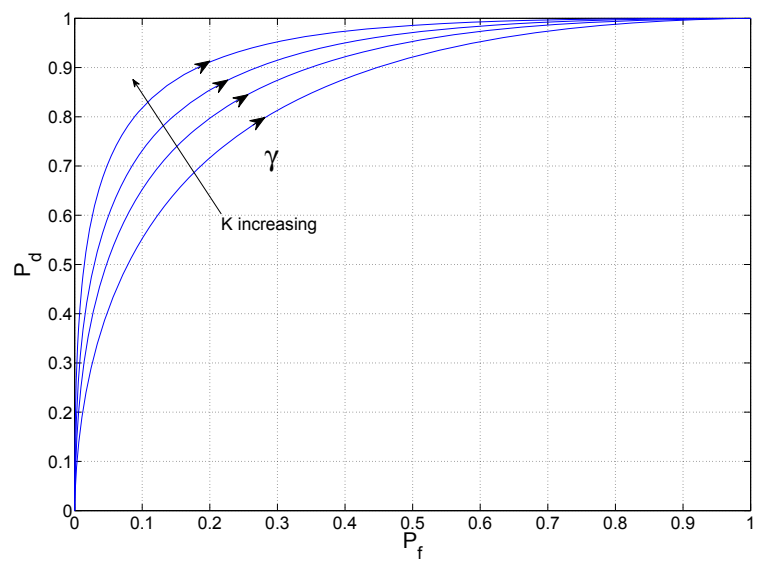

Fig. 3. ROC curve, $K$ and $\gamma$ dependency

The main idea of the adaptive detector block in Figure 1 is to use different threshold values for different number of observations received, $K$. The threshold values are obtained by solving the constraint optimization problem formulated in Section VI, and requires knowledge about the probabilistic channel distribution. The resulting adaptive detector is optimal 
in the sense of maximizing the expected value of the probability of detection, given a constraint on the expected value of the probability of false alarm.

\section{Preliminaries}

In this section, we present some of the results obtained in our previous work [14]. The results summarize the performance of NP detector, for DC level in WGN, with ideal, and Bernoulli communication channels. The equations presented here will be referred to, as an example, during our discussion about detector performance and optimal detector design in Sections V and VI, respectively. by:

The performance of the detector with ideal channel is given

$$
\begin{aligned}
& P_{f}=Q\left(\frac{\gamma}{\sqrt{\sigma^{2} / K}}\right) \\
& P_{d}=Q\left(\frac{\gamma-A}{\sqrt{\sigma^{2} / K}}\right) \\
& P_{d}=Q\left(Q^{-1}\left(P_{f}\right)-\sqrt{\frac{K A^{2}}{\sigma^{2}}}\right)
\end{aligned}
$$

where the error function $Q(x)=\int_{x}^{\infty} \frac{1}{\sqrt{2 \pi}} e^{-\frac{1}{2} t^{2}} d t$

With Bernoulli communication channel modeled by:

$$
p_{k}(K=k)=\left(\begin{array}{c}
N \\
k
\end{array}\right)(1-\lambda)^{k} \lambda^{(N-k)}
$$

where $\lambda$ is the channel drop rate. The degraded performance is expressed as:

$$
\begin{aligned}
E\left[P_{f}\right] & =\sum_{v=0}^{N}\left(\begin{array}{c}
N \\
v
\end{array}\right)(1-\lambda)^{v} \lambda^{(N-v)} Q\left(\frac{\gamma}{\sqrt{\sigma^{2} / v}}\right) \\
E\left[P_{d}\right] & =\sum_{v=0}^{N}\left(\begin{array}{l}
N \\
v
\end{array}\right)(1-\lambda)^{v} \lambda^{(N-v)} Q\left(\frac{\gamma-A}{\sqrt{\sigma^{2} / v}}\right)
\end{aligned}
$$

\section{DeteCtor PERformanCE: A PROBABILISTIC VIEWPOINT}

From Figure 3, it is clear that both $P_{d}$ and $P_{f}$ are functions of the number of received observations, $K$. Given the probabilistic description of $K$, it is easy to show that both $P_{d}$ and $P_{f}$ are random variables (transformations of the random variable $K$ ), defined on the sample space for $K$. To stress this fact, we denote the probability of detection for a specific realization of the random variable $K=k$ by $P_{d}^{k}(\gamma)$ (dependence on the threshold $\gamma$ is noted). Similarly, we define $P_{f}^{k}(\gamma)$ as the probability of false alarm for the realization $K=k$.

The fact that the performance metrics $P_{d}$ and $P_{f}$ are no longer deterministic is very important in the performance analysis and design of optimal detectors. We give an example to better illustrate the main idea.

Example 1. Equations (1) and (2) define the $P_{f}$ and $P_{d}$ as transformations for the random variable $K$. Figure 4 shows the distribution of $P_{f}$ and $P_{d}$, for $\gamma=0.1, A=0.2$, and $\sigma=1$. With an ideal channel, $K, P_{d}$, and $P_{f}$ are deterministic, and therefore, no probabilistic description is assigned to them.

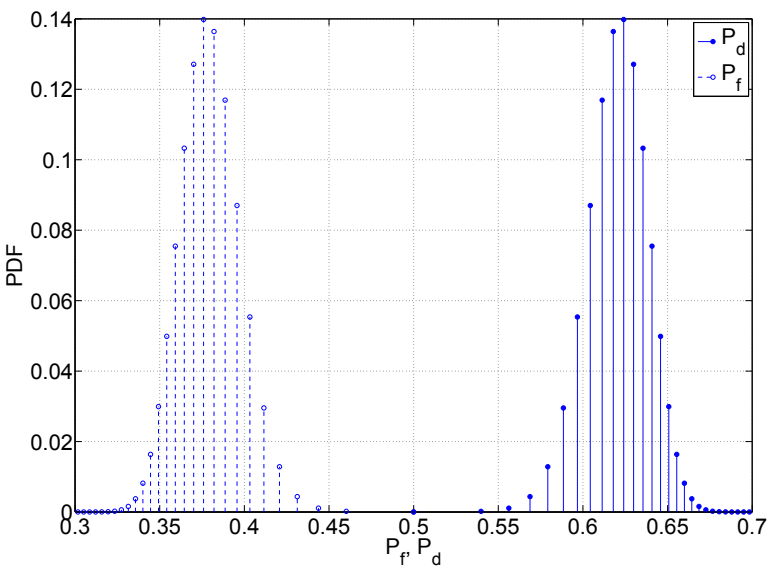

Fig. 4. $\quad$ PDF for $P_{f}$ and $P_{d}, \gamma=0.1$

Given this fact, the performance of the NP detector should be expressed in terms of the statistical properties of the distributions of the random variables $P_{f}$ and $P_{d}$. Therefore, optimizing the performance of the detector is done by modifying the distributions of $P_{f}$ and $P_{d}$. For example, by referring to Figure 4, we may seek to separate the two distributions by controlling their first and second order moments.

One of the parameters that could be adjusted to modify the distributions is the detector threshold, $\gamma$. As an example, we used the value $\gamma=0.1$ in Example 1 to produce the distributions in Figure 4. By using $\gamma=0.3$, we obtain Figure 5 .

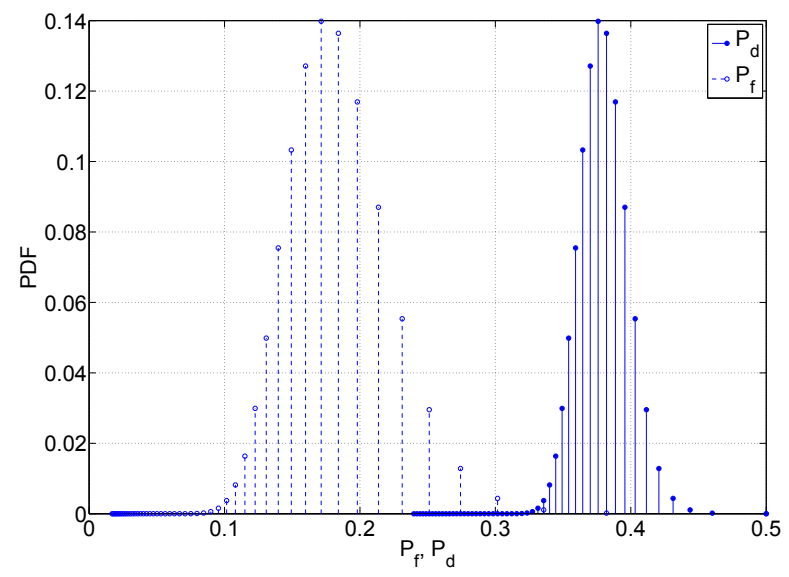

Fig. 5. $\quad$ PDF for $P_{f}$ and $P_{d}, \gamma=0.3$

Extending the idea of threshold adjustment, we can have more control on the distributions of $P_{d}$ and $P_{f}$ if we allowed individual thresholds to change. In other words, for every realization of the random variable $K=k$, we employ a different threshold $\gamma^{k}$ for the detector. In fact, this has the effect of controlling the transformations of the random variable $K$ to $P_{f}$ and $P_{d}$. Therefore, we express the probability of false alarm and the probability of detection as $P_{f}^{k}\left(\gamma^{k}\right)$ and $P_{d}^{k}\left(\gamma^{k}\right)$, 
respectively, noting that different values of the threshold $\gamma$ are used for different realizations of the number of samples received, $K$. This idea is used in the next section to obtain the optimal detector design.

\section{Vi. Optimal Detector Design}

The traditional optimal NP detector design is based on maximizing the probability of detection, $P_{d}$, subject to a constraint on the probability of false alarm, $P_{f}$, and by Neyman-Pearson Lemma [1], the optimal detector design (the critical region) is obtained.

With intermittent observations, the Neyman-Pearson Lemma defines the optimal detector design for a specific value of the observations received, $K=k$. In other words, the test optimizes the detector performance for a specific realization of the random variables $K, P_{d}$, and $P_{f}$. Since the probability of false alarm, $P_{f}$, is now a random variable, defining the constraint of the optimization problem in the form $P_{f}=\alpha$ is no longer applicable.

One natural choice for the statistical quantity to be optimized is the expected value for $P_{d}$ and $P_{f}$. Therefore, we seek to maximize the expected value of the probability of detection, subject to a constraint on the expected value of the probability of false alarm:

$$
\begin{aligned}
& \max E\left(P_{d}\right) \\
& E\left(P_{f}\right)=\alpha
\end{aligned}
$$

where:

$$
\begin{aligned}
& E\left(P_{f}\right)=\sum_{k=0}^{\infty} p_{K}(K=k) P_{f}^{k}\left(\gamma^{k}\right) \\
& E\left(P_{d}\right)=\sum_{k=0}^{\infty} p_{K}(K=k) P_{d}^{k}\left(\gamma^{k}\right)
\end{aligned}
$$

Since $P_{d}^{k}$ and $P_{f}^{k}$ are related by the ROC curve, we can assume the relationship has the functional form $P_{d}^{k}=f\left(P_{f}^{k}\right)$ Accordingly:

$$
\begin{aligned}
& E\left(P_{f}\right)=\sum_{k=0}^{\infty} p_{K}(K=k) P_{f}^{k}\left(\gamma^{k}\right) \\
& E\left(P_{d}\right)=\sum_{k=0}^{\infty} p_{K}(K=k) f\left(P_{f}^{k}\left(\gamma^{k}\right)\right)
\end{aligned}
$$

and the constraint optimization problem could be expressed as:

$$
\begin{aligned}
& \max _{\mathbf{P}_{\mathbf{f}}} E\left(P_{d}\right) \\
& E\left(P_{f}\right)=\alpha \\
& 0 \leq P_{f}^{k} \leq 1, k=\{0,1,2 \ldots .\}
\end{aligned}
$$

where $\mathbf{P}_{\mathbf{f}}$ is the vector of realizations of the random variable $P_{f}$ :

$$
\mathbf{P}_{\mathbf{f}}=\left[\begin{array}{lll}
P_{f}^{1}\left(\gamma^{1}\right) & P_{f}^{2}\left(\gamma^{2}\right) & \ldots
\end{array}\right]
$$

In practical applications, the vector $\mathbf{P}_{\mathbf{f}}$ cannot be with infinite dimension, since the probability of receiving a large number of samples, $P(K=k)$, in a finite duration $T$, approaches 0 as $K \rightarrow \infty$, for any practical channel.
This problem could be solved by using a Lagrangian multiplier method, as follows:

$$
\begin{aligned}
F & \left.=E\left(P_{d}\right)+\zeta\left[E\left(P_{f}\right)-\alpha\right)\right] \\
& =\sum_{K=0}^{K=\infty} p_{K}(K=k) P_{d}^{k}\left(\gamma^{k}\right)+\zeta\left[\sum_{K=0}^{K=\infty} p_{K}(K=k) P_{f}^{k}\left(\gamma^{k}\right)-\alpha\right]
\end{aligned}
$$

Differentiating with respect to $P_{f}^{k}$, and equating to zero, we get:

$$
\frac{d P_{d}^{k}\left(\gamma^{k}\right)}{d P_{f}^{k}\left(\gamma^{k}\right)}=-\zeta=\beta \quad k=0,1, \ldots \infty
$$

The constraint is expressed as:

$$
\sum_{K=0}^{K=\infty} p_{K}(K=k) P_{f}^{k}\left(\gamma^{k}\right)=\alpha
$$

Assuming that $k$ is upper bounded by $N$, which is the case for finite capacity channels, then Equation (9) represents $N$ equations in $P_{f}^{k}, \beta$, and together with the constraint in Equation (10), we can solve $N+1$ simultaneous equations in $P_{f}^{k}, \beta$, where $k=\{0,1,2 \ldots, N\}$. The thresholds are obtained using the inverse of the relationship $P_{f}^{k}\left(\gamma^{k}\right)$. The next example shows a practical application.

Example 2. Consider the problem of detecting DC level in WGN, presented in Section IV. Applying Equation (9) on Equation (3) and simplifying ${ }^{1}$, we get:

$$
P_{f}^{k}=Q\left(\sqrt{\frac{k A^{2}}{4 \sigma^{2}}}+\sqrt{\frac{\sigma^{2}}{k A^{2}}} \ln \beta\right)
$$

From Equations (4), (10), and (11) we get:

$$
\sum_{k=0}^{N}\left(\begin{array}{c}
N \\
k
\end{array}\right)(1-\lambda)^{k} \lambda^{(N-k)} Q\left(\sqrt{\frac{k A^{2}}{4 \sigma^{2}}}+\sqrt{\frac{\sigma^{2}}{k A^{2}}} \ln \beta\right)=\alpha
$$

Equation (12) could be solved numerically for $\beta$, and with substituting back for $\beta$ in Equation (11), we get $P_{f}^{k}$ for $k=$ $\{0,1,2 \ldots, N\}$. We get the threshold values from Equation (1):

$$
\gamma^{k}=\sqrt{\frac{\sigma^{2}}{k}} Q^{-1}\left(P_{f}^{k}\right)
$$

We give now some interpretations for the solution of the optimization problem. The left hand side of Equation (9) is the derivative of the ROC curve. So, this shows that the optimal solution is the set of points where the derivatives of the ROC curves (corresponding to different $K$ values) are equal. Since we may have infinite number of solutions, the one satisfying the constraint in Equation (10) results in the optimal solution.

Another interpretation for Equation (9) could be given. We have from the properties of ROC curves, that the derivative of the ROC curve at any point is equal to the threshold $\gamma$ (with respect to the original likelihood ratio, not the log ratio) [2]. This shows that the likelihood ratio test is still the optimal

\footnotetext{
${ }^{1}$ We used the fact that $\frac{d Q(x)}{d x}=\frac{1}{\sqrt{2 \pi}} e^{-x^{2} / 2}$ and $\frac{d Q^{-1}(x)}{d x}=$ $\sqrt{2 \pi} e^{\left[Q^{-1}(x)\right]^{2} / 2}$
} 
test in the case of intermittent observations, with the same fixed threshold (likelihood ratio threshold). However, if the likelihood ratio test results in a sufficient statistic (as in the case of DC level detection), the individual thresholds depend on the number of samples received, $k$ (as in Equation (13)).

In fact, the optimization problem seeks the most probable detector case (with respect to the number of samples received, $k$ ) and puts more weight on its performance, such that, on the average, the overall detection performance approaches its design objective. The adjustment in performance is achieved by varying the individual threshold, $\gamma^{k}$. On the other hand, if we have a very improbable $k$ value, then the probability of false alarm, $P_{f}^{k}\left(\gamma^{k}\right)$, for that case could be put arbitrarily large, without affecting the overall performance. This fact is easily shown by investigating Equation (7) and noting the weighting factors of $p_{k}(K=k)$.

Now the detector works as follows: Every time period, $T$, the detector decides on the state of nature, based on the number of observations received, $K=k$. For every value of $K$, there is a corresponding threshold value, $\gamma^{k}$, used by the detector for decision making (refer to Figure 1). The original detector design (with ideal channel), in terms of the test statistic, is not changed, but the threshold is adapted based on the observations received.

The results presented in the paper are general, and applicable to any NP detector, receiving a varying number of observations, as a result of the communication channel. In this paper, we explained the results on the reader decision. If there is a higher layer fusion center, collecting decisions from geographically spaced readers, then the analysis is applicable as well on the fusion center, since the decision fusion problem can be viewed as a two hypothesis detection problem with individual detector decisions being the observations [21].

\section{CONCLUSION}

In this paper we studied the optimal detector design, according to NP criterion of maximizing the probability of detection, given a constraint on the probability of false alarm, using wireless passive sensor networks, with intermittent observations. We have shown that the optimal design reduces to the likelihood ratio test, with a fixed threshold value, regardless of the number of observations received, $k$. We illustrated, by an example, the fact that although the optimal design does not change when a probabilistic channel exists, the detector implementation may need to be adapted, by adapting the threshold, to correctly implement the likelihood ratio test, for all values of the observations received, $k$.

As future work, we seek to optimize the detector performance with respect to other channel parameters. We are working also on the data fusion problem when there are multiple readers, reporting hard decisions to a fusion center. The overall system performance in this extended architecture is of interest. Other probabilistic channel models that capture the delay effect are also under research.

\section{ACKNOWLEDGMENT}

This work was supported in part by the National Science Foundation under Grant CNS-06152140, and NASA NRA NNX07AD12A.

\section{REFERENCES}

[1] S. M. Kay, Fundamentals of Statistical Signal Processing, Volume 2: Detection Theory, ser. Prentice Hall Signal Processing Series, A. V. Oppenheim, Ed. Prentice Hall PTR, 1998.

[2] T. K. Moon and W. C. Stirling, Mathematical Methods and Algorithms for Signal Processing. Prentice Hall, Upper Saddle River, NJ, 2000.

[3] J.-F. Chamberland and V. V. Veeravalli, "Wireless sensors in distributed detection applications," IEEE Signal Processing Magazine, 2007.

[4] B. Chen and P. K. Willett, "On the optimality of the likelihood-ratio test for local sensor decision rules in the presence of nonideal channels," IEEE Transactions on Information Theory, vol. 51, no. 2, pp. 693-699, Feb. 2005.

[5] B. Chen, L. Tong, and P. K. Varshney, "Channel-aware distributed detection in wireless sensor networks," IEEE Signal Processing Magazine, July 2006.

[6] S. Lewis, "A basic introduction to RFID technology and its use in the supply chain," LARAN RFID, Tech. Rep., 2004.

[7] H. Yoon, J. Xie, J. Abraham, V. Varadan, and P. Ruffin, "Passive wireless sensors using electrical transition of carbon nanotube junctions in polymer matrix," Smart Materials and Structures, Institute of Physics Publishing, UK, vol. 15, no. 1, 2006.

[8] P. Li and Y. Wen, "Design and fabrication of passive wireless sensor array system using composite coding resonant saw transducer." Measurement Science and Technology, Institute of Physics Publishing, UK, vol. 17, no. 2, 2006.

[9] F. Umbrecht, M. Wendlandt, D. Juncker, C. Hierold, and J. Neuenschwander, "A wireless implantable passive strain sensor system," IEEE Conference on Sensors, pp. 4 pp.-, Oct.-3 Nov. 2005.

[10] R. Matsuzaki and A. Todoroki, "Passive wireless strain monitoring of actual tire using capacitance-resistance change and multiple spectral features," Sensors and Actuators A: Physical, Elsevier B.V., vol. 126, no. 2,2005

[11] A. P. Sample, D. J. Yeager, P. S. Powledge, and J. R. Smith, "Design of a passively-powered, programmable sensing platform for UHF RFID systems," in IEEE International Conference on RFID, 2007.

[12] B. Chen, R. Jiang, T. Kasetkasem, and P. K. Varshney, "Channel aware decision fusion in wireless sensor networks," IEEE Transactions on Signal Processing, vol. 52, no. 12, pp. 3454-3458, Dec. 2004.

[13] R. Niu, B. Chen, and P. Varshney, "Fusion of decisions transmitted over rayleigh fading channels in wireless sensor networks," IEEE Transactions on Signal Processing, vol. 54, no. 3, pp. 1018-1027, March 2006.

[14] A. Tantawy, X. Koutsoukos, and G. Biswas, "Detection using intermittent observations for passive wireless sensors," American Control Conference, ACC2009, St. Louis, Missouri, 2009, to appear

[15] R. R. Tenney and N. R. Sandell, "Detection with distributed sensors," IEEE Transactions on Aerospace and Electronic Systems, vol. AES-17, no. 4, pp. 501-510, July 1981.

[16] G. Mergen and L. Tong, "Sensor-fusion center communication over multiaccess fading channels," IEEE International Conference on Acoustics, Speech, and Signal Processing, ICASSP 05, vol. 4, pp. iv/841-iv/844 Vol. 4, March 2005.

[17] M. Madishetty, V. Kanchumarthy, R. Viswanathan, and C. Gowda, "Distributed detection with channel errors," Proceedings of the ThirtySeventh Southeastern Symposium on System Theory, SSST '05., pp. 302306, March 2005.

[18] T. Duman and M. Salehi, "Decentralized detection over multiple-access channels," IEEE Transactions on Aerospace and Electronic Systems, vol. 34, no. 2, pp. 469-476, Apr 1998.

[19] W. Li and H. Dai, "Distributed detection of a deterministic signal in correlated gaussian noise over mac," IEEE International Symposium on Information Theory, pp. 2134-2138, July 2006.

[20] D. Bertsekas and R. Gallager, Data Networks. PRENTICE HALL, Englewood Cliffs, New Jersy, 1992.

[21] Z. Chair and P. Varshney, "Optimal data fusion in multiple sensor detection systems," Aerospace and Electronic Systems, IEEE Transactions on, vol. AES-22, no. 1, pp. 98-101, Jan. 1986. 